\title{
LOS DISCUTIDOS HALLAZGOS SUBACUÁTICOS DE ANFORAS ROMANAS DE LAS ISLAS CANARIAS
}

\section{THE CONTROVERSIAL UNDERWATER ROMAN AMPHORAE FINDS OF THE CANARY ISLANDS}

por

\author{
ESTHER ChÁvez ÁlVAREZ* y \\ ANTONIO TEJERA GASPAR ${ }^{* *}$
}

\begin{abstract}
RESUMEN En este artículo pretendemos hacer una revisión de los diferentes hallazgos arqueológicos de materiales cerámicos de supuesto origen romano de las Islas Canarias.
\end{abstract}

\begin{abstract}
In this article we have revised the underwater amphorae from Canary Islands but supposed roman origin.

Palabras claves Ánforas romanas, hallazgo subacuáticos, Islas Canarias.

Key words $\quad$ Roman amphorae, underwater, Canary Islands.
\end{abstract}

Entre los estudios arqueológicos que el prof. M. Pellicer trató en los años que ocupó la Cátedra de Arqueología de la Universidad de La Laguna (1968-1974), destacamos uno relacionado con el siempre discutido problema del conocimiento de las Islas Canarias en la Antigüedad, tema tan polémico como apasionante, del que hasta el momento presente, y a pesar de los avances en la investigación, nos encontramos aún con tantas dudas y problemas, como lo planteado por él en un trabajo de síntesis titulado "Ánforas de importación halladas en Canarias" (Pellicer 1970:43-56), en el que realizaba "una primera clasificación tipológica de las ánforas y anforoides descubiertos hasta ese momento" (Tejera y Balbín 1981: II; 1983: 9), y replanteaba de nuevo una cuestión tan debatida, manifestando que no era "ninguna novedad hablar sobre ánforas de importación halladas en Canarias. El Dr. Serra repetidas veces ha tratado esta cuestión desde 1965, cuando fue valorada la primera ánfora antigua de importación hallada y conocida en Canarias. Incluso bien poco más puede añadirse, porque a los dos anforoides ovoides últimamente hallados en Tenerife

* Profesora Asociada de Arqueología. Universidad de La Laguna

** Catedrático de Arqueología. Universidad de La Laguna 
no puede asignárseles una fecha exacta, porfalta de pruebas evidentes. No obstante creo que la importancia de la docena de hallazgos que ya se llevan hechos bien merece una comunicación de conjunto y de síntesis." (Pellicer 1970: 43-44).

Después de tres décadas de la aparición de ese artículo, y con ocasión del homenaje promovido por el Departamento de Prehistoria y Arqueología de la Universidad de Sevilla, hemos creído necesario hacer una revisión de todos esos hallazgos, que se han pretendido relacionar con la presencia del mundo romano en aguas del archipiélago canario.

La historia de los hallazgos subacuáticos en Canarias se remonta al año 1964, cuando se da a conocer por vez primera la existencia de una "ánfora fenicia" (Díaz 1964: 3), en aguas del sur de La Graciosa, que había sido hallada en una zona ubicada junto a la Caleta de Montaña Amarilla, en la "Playa de la Cocina". El ánfora fue descubierta por Jesús Urriza Echegaray, submarinista perteneciente a un grupo de pesca de Las Palmas de Gran Canaria, quien la encontró aislada, a unos $15 \mathrm{~m}$ de profundidad, y muy bien conservada. Fue depositada en el Museo de la Casa de Colón de Las Palmas de Gran Canaria (García y Bellido 1967: 29), y en la actualidad se halla en El Museo Canario de la misma ciudad.

Este primer hallazgo del que se tiene noticia (Fig. 1, $n^{\circ} 1 ;$ Fig. 2, $n^{\circ} 2-4$ ), propiciaría la aparición de una serie de artículos científicos, intentando asociar estos materiales cerámicos con la presencia de los romanos en estas islas. El prof. Serra Rafols, después de consultar a diversos especialistas en cerámicas antiguas, la daría a conocer en distintos foros científicos (Serra 1963-64: 231-233; 1965: 8; 1966a: 373-377), a la vez que lo hacía también A. García y Bellido, uno de los investigadores consultados, en una breve nota en donde manifestaba el interés de este descubrimiento (García y Bellido 1964: 8).

La valoración de esta primera ánfora descartó su adscripción fenicia, a la que se consideraría, en cambio, de época romana bajoimperial (Serra 1963-64: 231), y encontrando su mejor paralelo en la forma K109 del Ágora de Atenas (Robinson 1959: 68, pl. 15, p. 3.104), si bien se clasificará también como una posible Dressel 30 ó 33 y Pelichet 47 (García y Bellido 1967: 28; Pellicer 1970: 47; Blázquez 1977: 48), o Beltrán 74 (Beltrán 1970: 575 y 576, fig. 237, 2). Para E. Serra, según los datos suministrados por P. Cintas, se trataría de "un tipo de ánfora muy conocido en el Mediterráneo oriental y africano, cuyo origen será muy antiguo por su tradición, pero que se halla lo más a menudo con contexto tardo-romano de los siglos II a V de J. C. Su forma, a primera vista inusitada, se explica por no ser una pieza de transporte de géneros mercantiles, (...) sino un vaso de uso diario de los marineros; se fijaba por la punta en un hoyo de una tabla en la sobrequilla y, por las amplias espaldas, en una abertura del puente o entrepuente y así era fácil extraer con un cazo o achicador lo necesario de su contenido, como muestra un dibujo de Cintas, que acompaño" (Serra 1987: 427), (Fig. 2, n 1). Esta opinión se contrastaba con la de A. García y Bellido, quien la consideraba un indicio claro de comercio, probando así "... de una vez y de modo incontrovertible, la existencia de un tráfico entre las islas Canarias y el mundo romano" (García y Bellido 1964: 8). Para E. Serra, sin embargo, se trataba solamente de "un ánfora propia de los utensilios de a bordo de las naves antiguas" (Serra 1963-64: 232).

Al poco tiempo de producirse estos primeros descubrimientos, se tuvo noticia de otros dos hallazgos, uno de los cuales apareció en Arrecife (Lanzarote), en las inmediaciones de "Los Charcos", al norte de la "Playa de Bastián", localizado por D. Juan Fernández Coello, quien extrajo, a unos $6 \mathrm{~m}$ de profundidad, "un ánfora que aparecía totalmente recubierta de percebes y muy oxidada" (Topham 1965a: 6; 1965b: 8), de la que sólo pudo recuperarse la parte superior por estar adherida al fondo (Topham 1967: 4). El fragmento conservado, la parte correspondiente al cuello, asas y hombros (Fig. 1, n 3 ), actualmente en paradero desconocido, fue identificado como perteneciente a un ejemplar parecido al de La Graciosa (Serra 1967: 428; Jiménez 1966: 256), si bien en la última clasificación se relaciona con una Almagro 51C (Carandini et al. 1977: 144; Parker 1992: 96; Escribano y Mederos 1996c: 82). 
El segundo de estos descubrimientos tuvo lugar en aguas de La Graciosa, en la rada del "Rincón del Salado", muy cerca de la "Caleta del Sebo", siendo localizado por un pescador local, a $5 \mathrm{~m}$ de profundidad y a $150 \mathrm{~m}$ de la costa. Se trataba de una ánfora casi completa "de forma cónica y dos asas, de una altura aproximada de 55 centímetros" (Topham 1965c: 6; 1967:4), que más tarde sería adquirida por el Cabildo de Lanzarote para el futuro Museo Arqueológico (Fig. 1, $n^{\circ} 2$ ). Esta ánfora, idéntica en todo a la primera hallada en La Graciosa, salvo en el tamaño, ya que medía $10 \mathrm{~cm}$ menos, fue clasificada como una forma más pequeña de la K109 (Serra 1970: 428), o también como un "tipo 30 y 33 de Dressel y 47 de Pelichet y fechables en los siglos III y IV d. C"' (Pellicer 1970: 46)

Existen otras noticias de ánforas, al parecer similares, aunque no se conservan los hallazgos, de los que algunos fueron exportados al extranjero, según la información recogida por $\mathrm{S}$. Jiménez Sánchez, quien habla de "todo un pecio o resto de naufragio en la costa de Lanzarote, del que su inventor, un caballero extranjero, sustrajo cinco ánforas, según se dice" (Jiménez 1967: 199). Al mismo problema se refiere A. García y Bellido, quien aludiendo al hallazgo entre La Graciosa y Alegranza, dice que otra de ellas se la "... llevó un visitante extranjero" (García y Bellido 1967: 29). En un sentido similar se expresaría L. Diego Cuscoy, asegurando "... que otros descubrimientos de igual naturaleza han salido de las islas para el extranjero, y concretamente de la isla de Tenerife" (Diego Cuscoy 1967: 8). Estas circunstancias no nos han permitido conocer su forma, tipología, y menos aún su adscripción cultural.

Con posterioridad, se producirían dos nuevos hallazgos en aguas de Lanzarote y Tenerife. El de Lanzarote, fue dado a conocer por G. Topham, siendo su descubridor D. Manuel Tabares Cabrera. Fue localizado en el puerto de Arrecife, a poniente del islote de "Isla del Amor", o "Islote de Fermina", a unos $17 \mathrm{~m}$ de profundidad. Se trataba de un contenedor $\sin$ asas, de $67 \mathrm{~cm}$ de altura y 18 de diámetro máximo exterior, con una boca estrecha de $65 \mathrm{~cm}$, que fue clasificada por A. García y Bellido como romana, y datable entre los siglos II al IV d. C. (Topham 1967: 4), aunque con posterioridad, en un estudio más detallado, en el que además se corrige la altura -pasaba ahora a tener $39 \mathrm{~cm}$-, deja de considerarla romana y la fecha entre los siglos XV-XVI d. C. (García y Bellido 1970: 198-199).

El segundo hallazgo fue realizado por D. Valerio Jerez Veguero, y dado a conocer por L. Diego Cuscoy. Se encontró al norte de la Punta de Guadamojete, a unos $30 \mathrm{~m}$ de profundidad. Se trataba de un ejemplar de "poca altura de cuello, hombros más redondeados, carentes de as a y pico hueco y menos largo" (Diego Cuscoy 1967: 8), que también sería identificado como una ánfora romana de los siglos II y III d. C.

Con posterioridad, todos estos descubrimientos se relacionarían con los anforoides estudiados por $\mathrm{E}$. Borges García (1966: 378-394; 1970: 549-556; 1973: 703-708), los cuales eran muy frecuentes en distintos lugares de las costas atlánticas y mediterráneas, interpretándolos como "anforoides de iluminación" para colocarlos en las proas de las embarcaciones.

La colección de estos primeros anforoides se completaría con la aparición de otros análogos, hallados en Tenerife, como el recuperado en San Andrés (Serra 1970: 429), a 40m de profundidad; el de la Playa de Antequera, a $30 \mathrm{~m}$ de la costa y a 10 de profundidad; un fragmento encontrado frente al Real Club Náutico de Santa Cruz, a $10 \mathrm{~m}$ de la costa y a 6 de profundidad; y finalmente, dos de forma ovoide, uno en Roque Bermejo (Punta de Anaga), localizado a 20 m de profundidad, y otro en la Punta de Güímar, a 25m. A estos anforoides, en cambio, ya se les diferenciaba claramente de las tres primeras ánforas recuperadas en La Graciosa y Los Charcos, considerándolos "más bien modernos, del siglo XVII y probablemente fabricados en la zona de Cádiz" (Pellicer 1970: 46-47).

Si hasta ese momento la distribución de los descubrimientos subacuáticos se conocían sólo en La Graciosa, Lanzarote y Tenerife, la noticia del hallazgo de un tercer anforoide en aguas de Gran Canaria, frente a la Avenida Marítima, venía a ampliar el área de dispersión de estos hallazgos, aunque existía una referencia anterior sobre una anforita globular, localizada en aguas de la ciudad de Las Palmas de Gran Canaria, que había sido encontrada en 1968, a pesar de que desde el principio se plantearon ciertas reservas sobre su 
antigüedad (Jiménez 1967: 199). El anforoide de la Avenida Marítima, recuperado por un grupo de submarinistas de aquella ciudad, medía $43 \mathrm{~cm}$ de altura y fue encontrado a unos $40 \mathrm{~m}$ de la costa y a 9 de profundidad. Respecto de su valoración cultural se dijo ya en ese momento que "la primera impresión es que no se trata de una pieza de la Antigüedad Clásica, sino más bien de la época medieval o renacentista y acaso de origen árabe o hispano-portugués" (La Provincia 1975: 5).

El final de la década de los setenta, y el inicio de los ochenta, marca un punto de inflexión en cuanto a los hallazgos subacuáticos en Canarias, al pasár del estudio de descubrimientos casuales o fortuitos, a plantear de manera metódica y sistemática, una serie de prospecciones subacuáticas. Hasta ese momento los hallazgos conocidos se reducían a las 3 ánforas de La Graciosa y Lanzarote, y a 9 anforoides ( 1 de Lanzarote, 2 de Gran Canaria y 6 de Tenerife).

\begin{tabular}{|l|l|l|}
\hline \multicolumn{1}{|c|}{ Isla } & \multicolumn{1}{c|}{ Procedencia } & \multicolumn{1}{c|}{ Tipo } \\
\hline La Graciosa & Caleta de Montaña Amarilla, Playa de la Cocina & Ánfora $1^{\text {a }}$ K109 \\
\hline La Graciosa & Rincón del Salado, Caleta del Sebo & Ánfora 2 $^{\text {K } 109}$ \\
\hline Lanzarote & Los Charcos, al norte de Playa de Bastián & Ánfora Almagro 51C \\
\hline Lanzarote & Arrecife, “Isla del Amor" (Islote Fermina) & Anforoide \\
\hline Tenerife & Punta de Guadamojete & Anforoide \\
\hline Tenerife & San Andrés & Anforoide \\
\hline Tenerife & Playa de Antequera & Anforoide \\
\hline Tenerife & Frente a Real Club Náutico de Santa Cruz & Anforoide \\
\hline Tenerife & Roque Bermejo, Punta de Anaga & Anforoide \\
\hline Tenerife & Punta de Güímar & Anforoide \\
\hline Gran Canaria & Frente a Avenida Marítima & Anforoide \\
\hline Gran Canaria & Frente a la ciudad de Las Palmas de Gran Canaria & Anforoide \\
\hline
\end{tabular}

Tabla 1: Hallazgos subacuáticos hasta 1975 en aguas canarias (Elaboración propia)

A partir de esta fecha, A. Tejera y R. de Balbín, iniciarían una serie de prospecciones subacuáticas en las islas de Tenerife y Lanzarote, en las que proponían los siguientes objetivos: búsqueda de materiales romanos para corroborar las referencias de los textos clásicos relacionados con las islas; documentar las navegaciones a Canarias, anteriores al siglo XIV, así como el estudio, desde el punto de vista arqueológico, del tráfico comercial entre la Península y América a través de estas islas desde el siglo XV y en adelante (Tejera y Balbín 1981: II; 1983: 10).

En cuanto a Tenerife, isla en la que se había documentado un mayor número de hallazgos hasta ese momento, se hicieron prospecciones en la vertiente sureste, dentro del municipio de Santa Cruz de Tenerife, entre Roque Bermejo y Punta de Guadamojete. Para la realización de los trabajos se contaría con el apoyo técnico del Club de Buceo de Santa Cruz de Tenerife. Entre los materiales recuperados se encontraban la parte superior de una ánfora y un pivote, varios fragmentos de anforoides, un cuenco, una jarrita y un molino de mano (El Día 1980a: 3; 1980b: 3; García 1980: 3; Tejera y Balbín 1981: II; 1983: 10-13). Estos materiales, actualmente depositados en el Museo Arqueológico de Tenerife, se hallaron en diversos puntos de la costa, aislados y sin contexto arqueológico. El conjunto de materiales, a excepción del ánfora, correspondería a una época posterior al siglo XV, alcanzando en algunos casos hasta el XIX, e incluso a épocas más recientes (Tejera y Balbín 1983: 13). Del material recuperado merecen destacarse los anforoides que, según la 
clasificación de J. M. Goggin (1960: 3-37), abarcarían desde fines del S. XVI a mediados del S. XIX, correspondiéndose con las fases Media y Reciente de su clasificación, ya que se trataba de recipientes utilizados normalmente para el transporte de vino, vinagre, aguardiente, etc. en el comercio entre la Península Ibérica, Canarias y América.

Las prospecciones subacuáticas en Tenerife no se restringirán exclusivamente a las zonas conocidas por antiguos hallazgos, sino que en 1984 se afrontarán otros estudios en la vertiente norte y noreste de la isla, en la zona de El Prix, Roques de Anaga y Playa de Antequera (Delgado Baudet 1990: 34, nota 5). Estas primeras prospecciones se continuarían asimismo en diversos puntos del Archipiélago, concretamente en la isla de Fuerteventura, en Caleta de Fuste (Delgado 1985: 42; Muñoz 1995: 561); en La Gomera, en la Playa del Cabrito (Delgado 1985: 42; 1990: 34, nota 5); en la zona de El Río-La Graciosa, en la primera quincena de agosto de 1982, bajo la dirección de A. Tejera, M. Fernández-Miranda y R. de Balbín (Delgado 1990: 36; Miñano 1995: 132); y en la isla de Lanzarote, en torno a la zona de Los Charcos y el puerto de Arrecife en el año 1983, también dirigidas por A. Tejera y R. de Balbín (Miñano 1995: 133), en las que fueron recuperadas diversas piezas cerámicas.

Con posterioridad, desde 1985, los estudios de la arqueología subacuática en Canarias volverán a ser retomados por J. Delgado Baudet, quien realizaría dentro del Plan Nacional de Cartas Arqueológicas Subacuáticas del Ministerio de Cultura (Subdirección General de Arqueología), diversas Campañas de prospecciones en la Comunidad Autónoma de Canarias (Delgado 1987: 5; 1990: 36).

Estos trabajos se iniciarían en las islas de La Graciosa y Lanzarote, en las que se eligieron tres zonas relacionadas con las rutas de la navegación, ya conocidas desde antiguo por el hallazgo de diversos materiales, así como por las noticias de naufragios (Delgado 1987: 5). Las áreas prospectadas se centrarían en "El Río"-La Graciosa; en la "Bocayna", al sur y suroeste de Lanzarote, así como en Arrecife, al este y sureste de Lanzarote (Delgado 1990: 37). Aparte de esto, se estudiaría un conjunto de 134 piezas procedentes de hallazgos subacuáticos de las zonas prospectadas, que fueron depositadas en el Castillo de San Gabriel y en un almacén municipal de Arrecife (Lanzarote). El conjunto era de una gran variedad, ya que estaba compuesto por ánforas, anforoides, tinajas, jarros, bacines, cuencos, platos, palanganas, cantimploras, etc., catalogados todos como materiales "de tradición medieval o moderna, sincrónicos o posteriores a la conquista de Canarias" (Delgado 1987: 6).

Posteriormente, y continuando con estos trabajos, el grupo formado por J. M. Martín Herrera y A. M. Pérez Díaz, miembros del equipo de arqueología subacuática adscrito al Museo Arqueológico del Cabildo de Tenerife, solicitarían a la Viceconsejería de Cultura y Deportes del Gobierno de Canarias, la realización de un estudio específico en la zona de Radazul-Punta de Guadamojete, por ser el lugar en el que habían sido extraídos un mayor número de materiales (Pérez 1970: 237).

Por último, será G. Escribano quien retome, a partir de la década de los noventa, la investigación subacuática en Canarias con la realización del Proyecto de Investigación, "Catalogación e inventario de la Arqueología Submarina en Canarias" (1993), así como su Memoria de Licenciatura, "La Arqueología subacuática en Tenerife" (1995), dirigida por A. Tejera Gaspar. El resultado de estas investigaciones será dado a conocer en diversas publicaciones en las que se recopilan los hallazgos anfóricos romanos en Canarias (Escribano y Mederos 1996a: 42-47; 1996c: 75-98), así como los anforoides (Escribano y Mederos 1998: 539-568), dando a conocer asimismo algunos inéditos. Estos trabajos se completarán con una serie de prospecciones de urgencia, realizadas en el puerto de Arrecife, en Lanzarote; en la playa de El Burrero de Carrizal, Ingenio, Gran Canaria, así como en la playa de Vallehermoso, La Gomera (Escribano y Mederos 1996b: 209), siendo estas dos últimas ampliadas en 1995 con una serie de sondeos (Escribano et al. 2000a: 361-381; 2000b: 383-397), en las que se documentaron restos de pecios de los siglos XVII-XVIII.

Llegados a este punto, hemos de decir que con relación a las ánforas a las que se les ha atribuido un origen romano, aparte de las tres primeras (Fig. 1), dadas a conocer en la década de los sesenta, el desarrollo 
de las prospecciones subacuáticas posteriores ha aumentado su número hasta alcanzar las 16 ánforas (Fig. 3), que se distribuyen por las islas del modo siguiente:

\begin{tabular}{|l|l|c|c|c|}
\hline \multicolumn{1}{|c|}{ Isla } & \multicolumn{1}{|c|}{ Depósito } & Tipo & $\mathrm{N}^{\circ}$ y piezas & Doc. \\
\hline La Graciosa & Museo Canario, Las Palmas & Ágora K109 & $\mathrm{n}^{\circ} 1-1$ & Foto/dib. \\
\hline Lanzarote & Desconocido & Almagro 51C & $\mathrm{n}^{\circ} 2-1$ & Foto \\
\hline La Graciosa & Desaparecida del Cabildo de Lanzarote & Ágora K109 & $\mathrm{n}^{\circ} 3-1$ & - \\
\hline La Graciosa & Desconocido. Exportada ilegalmente al extranjero & $?$ & $\mathrm{n}^{\circ} 4-1$ & - \\
\hline Lanzarote & Desconocido. Exportadas ilegalmente al extranjero & $?$ & $\mathrm{n}^{\circ} 5$ a 9-5 & - \\
\hline Tenerife & Museo Arqueológico, Santa Cruz de Tenerife, $\mathrm{n}^{\circ}$ 1069 & Dressel 2-4 & $\mathrm{n}^{\circ} 10-1$ & Foto/dib. \\
\hline La Graciosa & Museo Sánchez Araña, Santa Lucía de Tirajana & Ágora K109 & $\mathrm{n}^{\circ} 11-1$ & Foto/dib. \\
\hline La Graciosa & Almacén Municipal de Arrecife, Lanzarote & Dressel 7-11 & $\mathrm{n}^{\circ} 12$ & Foto/dib. \\
\hline Tenerife & Museo Arqueológico, Santa Cruz de Tenerife, $\mathrm{n}^{\circ} 1084$ & Benghazi MR & $\mathrm{n}^{\circ} 13$ & Foto/dib. \\
\hline Tenerife & Colección particular de Tenerife & Africana II & $\mathrm{n}^{\circ} 14$ & Foto/dib. \\
\hline Gran Canaria & $?$ & Pascual 1 & $\mathrm{n}^{\circ} 15$ & - \\
\hline Fuerteventura & $?$ & Dressel 30 & $\mathrm{n}^{\circ} 16$ & Foto \\
\hline
\end{tabular}

Tabla 2: Hallazgos de ánforas con atribución romana en aguas canarias (Fuente: Elaboración propia a partir de Escribano y Mederos 1996a y 1996c)

Si desglosamos la muestra, hemos de señalar, en primer lugar, que de un total de 16 ánforas, seis fueron exportadas ilegalmente al extranjero ( $\mathrm{n}^{\circ}$ 4-9), no teniendo de ellas ningún dato, salvo esa referencia (Jiménez 1967: 199; García y Bellido 1967:29), por lo que nos parece demasiado arriesgado incluirlas como romanas, ya que por una parte existía una gran confusión sobre su atribución en el momento en el que comienzan a aparecer, y, de otra, dado que los anforoides son los hallazgos más comunes en aguas de Canarias, cabría pensar que más bien pudieran tratarse de este tipo de recipientes.

En segundo lugar, de las diez restantes, existe una duplicación para el caso de las clasificadas como Ágora K $109\left(n^{\circ} 1,3\right.$ y 11 de la Tabla 2 y Fig. 3), de la que se presentan tres ejemplares, aunque en la actualidad sólo se conocen dos, uno depositado en el Museo Canario de Las Palmas de Gran Canaria (Ánfora $n^{\circ}$ 1, Fig. 1, $\mathrm{n}^{\circ}$ 1), y otro en esta misma isla, en el Museo Sánchez Araña de Santa Lucía de Tirajana (Ánfora $n^{\circ} 11$, Fig. $\left.1, n^{\circ} 2\right)$. Esta confusión se produce porque el ánfora $n^{\circ} 3$, si bien procede de La Graciosa, fue adquirida por el Museo Sánchez Araña en la década de los ochenta (Ánfora n 11 ), como se puede comprobar por las fotos de su publicación, de modo que si las comparamos con las primeras (Topham 1965c: 6; Jiménez 1966: 256; García y Bellido 1967: 17; Ídem 1970: lám. I), podemos comprobar que se trata de la misma que se recoge posteriormente, como expuesta en el Museo Sánchez Araña (Atoche et al. 1995: 70; Escribano y Mederos 1996a: 45). No se trataría en ese caso de una ánfora inédita, según G. Escribano y A. Mederos (1996c: 83-84), sino que probablemente se debe a un error en la contrastación de la documentación.

Por otro lado, y respecto a esta última ánfora hay que añadir que los resultados de los análisis petrográficos realizados recientemente por el equipo que ha estudiado el yacimiento lanzaroteño de El Bebedero (muestra LG94), aportan la siguiente conclusión, en el sentido de que "no permiten establecer una correlación con ninguno de los análisis recogidos por Peacocky Williams ni con los efectuados en las ánforas de la Colonia Celsa (Velilla de Ebro-Zaragoza)", por lo que "su lugar de fabricación es incierto, aunque por los componentes volcánicos que contiene la pasta hay que descartar un origen español", lo que les lleva a "encuadrarla 
en el Renacimiento, momento en el que resurgen los modelos clásicos y se hacen frecuentes las imitaciones" (Atoche et al. 1995: 68 y 71). No obstante, esta opinión es refutada al considerar que "los componentes volcánicos que caracterizan la lámina delgada (LG94) (...) coinciden con la procedencia que sostenemos para las K-109, el Mar Egeo, puesto que en dicha región existen rocas volcánicas en las Cícladas (Melos, Kimolos, Poliagos, Tera), Psara, Lesbos, Ayios Efstratios, Limnos, Samotracia, Eubea, Beocia, Egina y en áreas puntuales de la Argolide y Laconia" (Mederos y Escribano 1997: 229). De cualquier forma, habría que recordar que los análisis de D. P. S. Peacock y D. F. Williams no recogen todas las ánforas romanas conocidas, sino las más comunes (Peacock y Williams 1991: 79). Estas reservas sobre la segura adscripción romana de algunos de estos hallazgos ya había sido puesta de manifiesto por A. García y Bellido, quien refiriéndose a las dos ánforas halladas en La Graciosa, (Ágora K109) decía que "no se han aportado aún pruebas fehacientes y concluyentes de que esta datación sea indiscutiblemente exacta; vale como decir que cabrian dudas de que el ánfora de isla Graciosa fuera realmente un producto romano de hacia los siglos III o IV de la Era." (García y Bellido 1970: 194).

De las 16 ánforas de las que partíamos, hemos de reducir su número a 9. De éstas, habría que tener en cuenta varias cuestiones, sobre todo en lo que se refiere al ánfora $\mathrm{n}^{\circ} 2$ (Almagro $51 \mathrm{C}$ ), de la que, como hemos dicho, fue localizada al norte de Arrecife, en las inmediaciones de Los Charcos, si bien actualmente se encuentra en paradero desconocido, aunque se poseen dos fotos publicadas en blanco y negro, una correspondiente al momento de su hallazgo (Topham 1965b: 8) y otra (Fig. 1, $\mathbf{n}^{\circ}$ 3) hecha con posterioridad (Serra 1970: fig. 2). De ella se ha presentado recientemente un dibujo (Escribano y Mederos 1996c: 93, fig. 2), que ha sido utilizado para su clasificación. El hecho de que el ánfora se halle desaparecida, nos obliga a preguntarnos cómo pudo realizarse aquél (Fig. $3, \mathrm{n}^{\circ} 2$ ), a no ser que se trate de un calco de la foto publicada por $\mathrm{E}$. Serra. En efecto, si superponemos un calco a su cliché, se puede comprobar que ambos coinciden al tener la misma escala. Este procedimiento no lo consideramos de lo más adecuado para presentar y clasificar una pieza, pues, aún cuando se consiga un perfil aproximado, faltaría su sección, en la que se observaría con detalle el grosor de las paredes, la forma de la boca y el labio, que en la mayoría de las ocasiones resulta fundamental para una correcta clasificación. Por otro lado, nos faltan también una serie de detalles que no siempre puede aportar una foto en blanco y negro, como la técnica de fabricación, que suponemos a torno, así como las características de la pasta, que resultan muy importantes para asignarle una procedencia, tal y como ha sido señalado por $\mathrm{P}$. Atoche, quien refiriéndose a esta misma pieza dice que "en cualquier caso, para confirmar esas supuestas atribuciones sería conveniente analizar directamente las piezas y, si es posible, efectuar análisis petrográficos. También sería determinante la localización de los pecios y su estudio arqueológico" (Atoche et al. 1995: 76).

Otra de las ánforas localizada en aguas de Lanzarote es la $n^{\circ} 12$, clasificada recientemente como una Dressel 7-11 (Escribano y Mederos 1996c: 84). Si nos fijamos en esta pieza, de la que existen varias fotos como documentación gráfica (Delgado 1987: 7, fig. 9; Ídem 1990: 43, fig. 3), así como un dibujo(Escribano y Mederos 1996c: 96, fig. 5), se puede observar que sólo se conserva parte del cuello, las asas, e inicio del hombro, faltándole, en cambio, la boca y el labio (Fig. 3, n 12), lo que hace muy dudosa su clasificación, así como su atribución cultural, que bien pudiera encontrarse dentro de los tipos propios de época bajomedieval o moderna.

Igualmente, para el caso de la isla de Tenerife se han documentado tres piezas (ánforas $n^{\circ} 10,13$ y 14 de la Tabla 2), de las que sólo hemos podido trabajar con las dos primeras, actualmente en los fondos del Museo Arqueológico de Santa Cruz de Tenerife ( $\mathrm{n}^{\circ}$ de registro 1.069 y 1.084) ${ }^{1}$, puesto que la tercera pertenece a una colección particular que no hemos podido consultar. Así, en cuanto al ánfora $n^{\circ} 10$, clasificada como

1. Desde estas páginas queremos agradecer todas las facilidades dadas para consultar este material, tanto a su director, $R$. González Antón, como a diferentes miembros de la Institución. 
Dressel 2-4 (Escribano y Mederos 1996c: 83 y 94, fig. 3), podemos comprobar que tanto su dibujo como su clasificación no resultan muy precisos. Si comparamos los dibujos (Fig. 3, n 10 y Fig. 4, no 1 ) se observa, en primer lugar, cómo la parte conservada de la pieza es mucho mayor ${ }^{2}$. En segundo lugar, presenta una boca acampanada con borde exvasado y labio doblemente moldurado, cuello cilíndrico con leves acanaladuras en la parte inferior, flanqueado por asas cortas que no son acodadas, de sección oval y con resalte central en la cara externa, que se unen a la boca montando sobre la moldura inferior del labio. En la parte inferior las asas se unen a la pared a la altura del hombro, que no es carenado, como sugiere el paralelo que se presenta de una Dressel 2-4 (Escribano y Mederos 1996c: 94, fig. 3). Por tanto, todo ello nos indica que una clasificación más aproximada de la pieza la englobaría en todo caso, dentro del grupo de las Dressel 7-11, más concretamente con una Dressel 10, teniendo en cuenta que existen en ella ciertas variantes como el asa que monta sobre la moldura inferior del labio, cuando lo normal es que arranque por debajo de la boca, así como el que presente un cierto estrechamiento en la parte inferior donde monta sobre el hombro, producto probablemente de la presión ejercida por el alfarero en el momento de pegar el asa al cuerpo del ánfora.

De igual forma, el ánfora $\mathrm{n}^{\circ} 13$ (Fig. $3, \mathrm{n}^{\circ} 13$ y Fig. $4, \mathrm{n}^{\circ}$ 2), clasificada como una Benghazi MR1, es una pieza pequeña, de la que se conserva boca, cuello, asas y gran parte del cuerpo ${ }^{3}$. Si observamos con detenimiento los dibujos, advertiremos en primer lugar, que la pieza presenta un cuello cilíndrico con estrías al interior, $y$ al exterior a diferente altura, rematado con un labio exvasado en lugar de entrante, como es común en los paralelos conocidos (Scilliano y Sibella 1991: Ágora M254). Además, las asas de sección oval apoyan directamente sobre los hombros, justo encima de la carena, mientras que en todos los paralelos conocidos, lo hacen a la mitad del hombro, sin alcanzar nunca la altura de la carena, y cuya sección es de tendencia circular. En cualquier caso, de tratarse de un ánfora romana de la Tripolitana, estaríamos ante una variante no conocida de la misma, si bien también podría tratarse de una botija, como las identificadas por F. Amores y N. Chisvert en rellenos de bóvedas sevillanas de época bajomedieval y moderna (Amores y Chisvert 1993: 311, fig. 88 P).

Finalmente, a estas siete piezas habría que añadir dos más, de las que contamos con muy pocos datos, pues sólo conocemos su procedencia de Gran Canaria y Fuerteventura, sin más precisión; y su clasificación, Pascual 1 y Dressel 30, respectivamente, si bien para el caso de la de Fuerteventura, contamos al menos con una foto (Escribano y Mederos 1996a: 46-47), mientras que para la de Gran Canaria sabemos, indirectamente, que se trata de una pieza completa (Mederos y Escribano 1997: 234). Por tanto, sería necesario contar con otro tipo de datos, como una buena descripción macroscópica, dibujo, etc., que resultan imprescindibles para una valoración adecuada de estos materiales.

Hemos de decir por último, que desde la década de los sesenta, en que comienzan los hallazgos subacuáticos en las Islas Canarias, hasta la actualidad, se han dado a conocer como romanas unas 16 ánforas en aguas canarias que, como hemos analizado en párrafos anteriores, quedarían reducidas sólo a nueve, y algunas de ellas bastante dudosas, tanto por la adscripción cultural como por la clasificación propuesta. Todas proceden de hallazgos subacuáticos aislados, carentes de contexto arqueológico, por lo que sería necesario una revisión en detalle, comenzando por su descripción y dibujo, así como la localización y estudio arqueológico de los "posibles pecios hundidos", ya que hasta este momento no se ha relacionado ninguna de las ánforas con los correspondientes pecios, seguramente por las propias dificultades para su hallazgo en los litorales

2. La pasta es de color ocre (D66), dura, de factura rugosa, con algunas vacuolas y pequeños desgrasantes oscuros. De ella sólo se conserva borde, cuello, asas y parte del hombro. Sus medidas son: $14 \mathrm{~cm}$. $\emptyset$ boca, 22'3 cm de altura máx. conservada y 28 ' $5 \mathrm{~cm}$ de anchura máx. conservada. No presenta marcas de ningún tipo. Para los colores hemos utilizado el Code Expolaire de A. Cailleux y G. Taylor (1963).

3. La pasta es de color tierra verde tostada (C34), muy dura, de factura rugosa, con pequeñas vacuolas y desgrasantes oscuros, rojizos y brillantes. Sus medidas son: $6 \mathrm{~cm}$. $\emptyset$ boca, $277^{\prime} \mathrm{cm}$. de altura máx. conservada y $20^{\prime} 5 \mathrm{~cm}$. de anchura máx. conservada. No presenta marcas de ningún tipo. 
canarios, ya que a excepción de algunas zonas de Lanzarote y Fuerteventura, que poseen una mayor plataforma costera, en las islas restantes, por el contrario, es prácticamente inexistente, ya que aparece muy pronto una pendiente muy acusada que junto a la fuerte acción de las mareas y corrientes hacen que los pecios y sus materiales sean rápidamente esparcidos y arrastrados a las profundidades de los fondos marinos.

Entre los múltiples problemas existentes sobre las ánforas pretendidamente romanas halladas en las Islas Canarias desde la década de los años sesenta del siglo XX, el más destacado, sin duda, es, como hemos dicho, la falta de contextos arqueológicos claros de época romana que apoyen este origen para los materiales analizados. Las ánforas han sido clasificadas como romanas a partir de sus parecidos formales, al no existir en ellas ninguna otra evidencia que permita tal adscripción, porque no poseen ninguna marca de propietario, o de contenido, como suele ser frecuente en este tipo de envases. Lo más relevante, sin embargo, es que no se ha documentado hasta ahora ningún otro material típicamente romano, ya se trate de cerámicas finas (campanienses, sigillatas), o comunes, mientras que, por el contrario, los materiales "asociados" a estas ánforas, entre los que son más comunes los anforoides, todos pertenecen a un período que abarca los siglos XVI al XIX, lo que nos obliga a ser prudentes a la hora de valorar estos datos como de segura procedencia romana, hasta tanto no podamos contar con ejemplares tipológicamente bien definidos y caracterizados (Chávez 1998: 43), así como con una información arqueológica complementaria más sólida.

Nuestras reservas con relación al posible origen romano de los materiales analizados, es una consecuencia de las circunstancias en las que se han producido estos hallazgos, así como de los problemas formales que nos plantean estas ánforas, de las que no cabe desechar una posible pervivencia en épocas posteriores a partir de formas romanas precedentes, y sin que tampoco nosotros rechacemos totalmente una adscripción romana para algunas de ellas.

El conocimiento seguro que los romanos tuvieron de estas islas (Delgado Delgado 1993: 74), se remontaría al 82-81 a. C., basándose en la obra "Vidas Paralelas" de Plutarco (1/2 s. I-inic. S. II d. C.), quien en un pasaje referido a la vida de Sertorio (Plu., Sert., 8, 1-2 y 9, 1) habla de la existencia de dos islas en el Atlántico, texto considerado por muchos autores, como un dato preciso sobre su descubrimiento en el primer cuarto del siglo I a. C., aunque es, sobre todo, a través de la descripción de las "Islas Afortunadas" del texto de Plinio (Plin., HN, VI, 202-205), como se confirma su conocimiento por parte de los romanos.

Por todo lo anteriormente expuesto queremos finalmente señalar, que con este trabajo no hemos pretendido cerrar tema tan debatido, sino bien al contrario, dejarlo abierto a la discusión debido a los múltiples problemas que quedan aún por resolver.

\section{BIBLIOGRAFÍA}

BELTRÁN LLORIS, M. (1970): Las ánforas romanas en España. Zaragoza.

BLÁZQUEZ, J. Ma (1977): "Las Islas Canarias en la Antigüedad", Anuario de Estudios Atlánticos 23: 35-50.

BORGES GARCÍA, E. (1966): “Anforetas de iluminaçao de embacaçoes romanas, encontradas na costa portuguesa”, IX Congreso Nacional de Arqueología (Valladolid, 1965): 378-394.

- (1970): "Nuevos estudios sobre anforetas encontradas en las costas e islas atlánticas y mediterráneas", XI Congreso Nacional de Arqueología (Mérida, 1968): 549-556.

(1973): "Noticia muy actual sobre anforetas", XII Congreso Nacional de Arqueología (Jaén, 1971): 703-708.

CABRERA, J. C., PERERA, Ma A. y TEJERA, A.(1999): Majos. La primitiva población de Lanzarote. Islas Canarias. Madrid.

CARANDINI, A.; PANELLA, C. et alii (1977): Ostia IV. Studi Miscelanei 23. Roma. 
CHÁVEZ Ál VAREZ, Ma E. (1998): "Arqueología romana", Enciclopedia de Patrimonio Histórico de Canarias. Lanzarote y Fuerteventura: 40-43. Gobierno de Canarias. Santa Cruz de Tenerife.

DELGADO BAUDET, J. (1985): “Arqueología Submarina en Canarias”, Revista de Arqueología 56: 40-45. (1987): “Arqueología Subacuática en Canarias”, Revista de Arqueología 70: 5-7.

(1990): "La actividad arqueológica subacuática en Canarias", Investigaciones Arqueologicas en Canarias II: 31-45.

DELGADO DELGADO, J. (1993): "De Posidonio a Floro: Las Insulae Fortunatae de Sertorio", Revista de Historia Canaria 177: 61-74.

DÍAZ, M. (1964): "Interesante hallazgo arqueológico en La Graciosa", Diario de Las Palmas 5/10/1964: 3.

DIEGO CUSCOY, L.: "Un ánfora romana en aguas de Tenerife", El Día 31-10-1967: 6 y 8.

EL DÍA (1980a): "Hallazgos de cerámica romana en el mar de Tenerife", El Día 15-01-1980: 3. (1980b): "Más hallazgos de arqueología submarina”, El Día 1-05-1980: 3.

ESCRIBANO COBO, G. (1995): La Arqueología subacuática en Tenerife. Memoria de Licenciatura inédita. Universidad de La Laguna.

ESCRIBANO COBO, G. y MEDEROS MARTÍN, A. (1996a): “Canarias. Límite meridional en la periferia del Imperio Romano", Revista de Arqueología 184: 42-47.

(1996b): "Balance y nuevas perspectivas de la arqueología submarina en las Islas Canarias", Cuadernos de Arqueología Marítima 4: 203-215.

__ (1996c): “¿Ánforas romanas en las Islas Canarias?. Revisión de un aparente espejismo histórico”, Tabona IX: 75-98.

_ (1998): "Botijas en yacimientos arqueológicos subacuáticos de las Islas Canarias. Una fuente complementaria para el análisis del comercio canario-americano", XII Coloquio de Historia CanarioAmericano I: 539-568.

ESCRIBANO, G.; MEDEROS, A. y CHINEA, D. (2000a): "Prospección y sondeo subacuático en la Playa de El Burrero (Ingenio, Gran Canaria, Islas Canarias)", Investigaciones Arqueológicas en Canarias 6: 361-381.

- (2000b): "Prospección y sondeo subacuático en la playa de Vallehermoso(La Gomera, Islas Canarias)", Investigaciones Arqueológicas en Canarias 6: 361-381.

GARCÍA, J. L. (1980): "Las “ánforas romanas" son del siglo XVI y llevaban aceitunas a América”, Diario de Avisos 16-01-1980: 3.

GARCÍA y BELLIDO, A. (1964): "Las Canarias en la Antigüedad”, ABC 5-12-1964: 8

(1967): Las Islas Atlánticas en el Mundo Antiguo. Las Palmas de Gran Canaria.

__ (1970): "Sobre las ánforas antiguas de Canarias", Homenaje a Elías Serra Rafols vol. 2: 193-199. Universidad de La Laguna, La Laguna.

GOGGIN, J. M. (1960): “The Spanish Olive Jar. An Introductory Study”, Yale University Publications in Anthropology 6: 3-37.

JIMÉNEZ SÁNCHEZ, S. (1966): "Más ánforas romanas en aguas lanzaroteñas", Revista de Historia Canaria XXX: 255-257.

- (1967): "Hallazgos submarinos", Revista de Historia Canaria XXXI: 198-200.

LA PROVINCIA (1975): “Ánfora antigua aparecida en la Avenida Marítima”, La Provincia 18-05-1975: 5.

MARTÍN DE GUZMÁN, C. (1977): "Arqueología submarina”, Historia General de las Islas Canarias de Agustín Millares Torres, Tomo III: 24-25. Las Palmas.

MEDEROS MARTÍN, A. y ESCRIBANO COBO, G. (1997): "Una etapa en la ruta Mogador-Canarias: cerámica romana en Lanzarote y su relación con hallazgos submarinos", Spal 6: 211-242.

MIÑANO DOMÍNGUEZ, A. I. (1995): "Inventario de yacimientos arqueológicos marítimos y litorales de la Provincia de Las Palmas", Cuadernos de Arqueología Marítima 3: 125-157. 
MUÑOZ AMEZCUA, J. M. (1995): "Los grabados naviformes de Tijonay", IV Jornadas de Estudios sobre Lanzarote.y Fuerteventura II: 555-616.

PARKER, A. J. (1992): Ancient Shipwrecks of the Mediterranean \& the Roman Provinces. B.A.R. I.S. 580. Oxford.

PELLICER, M. (1970): “Ánforas de importación halladas en Canarias”, Estudios Canarios XIV-XV:43-56.

ROBINSON, H. S. (1959): Pottery of the Roman Period. Chronology. The Athenian Agora V. Princenton.

SERRA, E. (1963-64): "La primera ánfora romana hallada en Canarias", Revista de Historia Canaria XXIX: 231-233.

(1965): "La primera ánfora romana hallada en Canarias", El Eco de Canarias 12-02-1965: 8.

(1966a): "Ánfora antigua en Canarias", IX Congreso Nacional de Arqueología (Valladolid, 1965): 373-377.

- (1966b): "Actividades de la Delegación de zona del Distrito Universitario de La Laguna. Año 1965", Noticiario Arqueológico Hispánico VIII-IX: 294-297.

-(1970): "Más cerámicas antiguas en aguas de Canarias", XI Congreso Nacional de Arqueología (Mérida, 1968): 428-430.

(1987): "Una ánfora romana en Canarias", en G. Arias, Repertorios de caminos de la Hispania romana: 427-428. Madrid. Publicado originalmente en el Miliario Extravagante 8, (marzo, 1965).

TEJERA, A. y BALBÍN, R. de (1981): “Arqueología submarina en Tenerife”, Diario de Avisos 17-10-1981: II (Suplemento).

(1983): "La arqueología submarina en Tenerife", El Museo Canario XLIII: 9-17.

TOPHAM, G. (1965a): "Hallazgo de un ánfora, que puede pertenecer a la época de las invasiones del morato Arraez (1586), Antena 19-01-1965: 6.

(1965b): "Otra ánfora, antiquísima, descubierta en aguas de la isla", El Eco de Canarias 21-01-1965: 8. (1965c): "Una ánfora, posiblemente de origen romano, localizada y extraída en aguas de La Graciosa", El Eco de Canarias 19-12-1965: 6.

(1967): "Importante descubrimiento arqueológico en los fondos marinos de Arrecife", La Tarde 27-031967: 4 . 

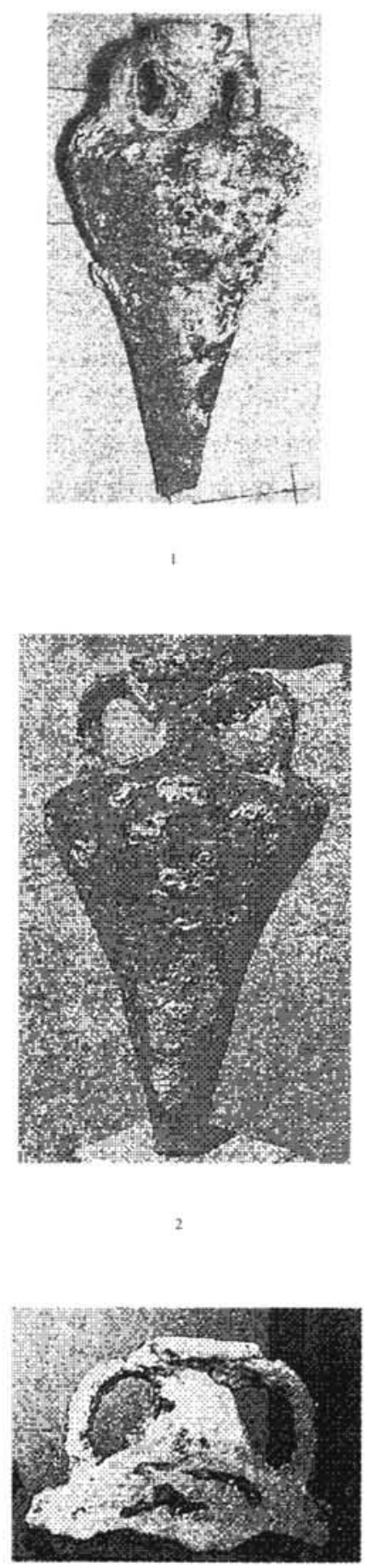

Figura 1. Fotos de las tres primeras ánforas aparecidadas en aguas canarias. 1: $1^{a}$ ánfora de La Graciosa (García y Bellido 1967: fig. 1); 2: 2ª́nfora de la La Graciosa (García y Bellido 1967: fig. 2); y 3: Ánfora de Los Charcos, Arrefice, Lanzarote (Serra 1970: fig. 2). 

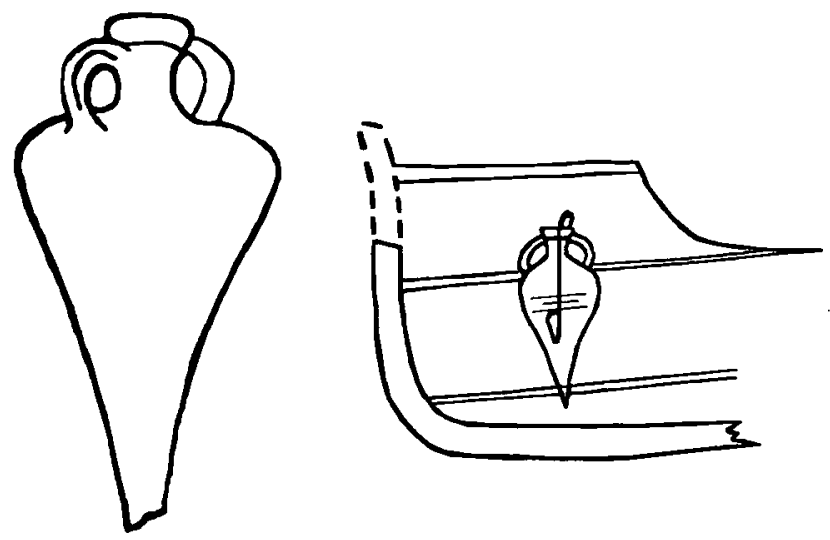

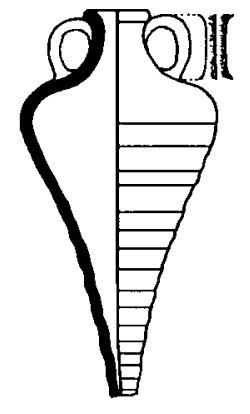

2

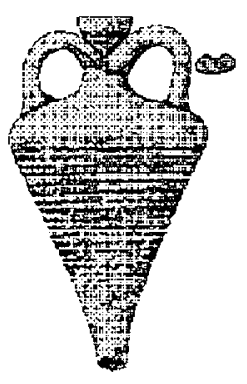

3

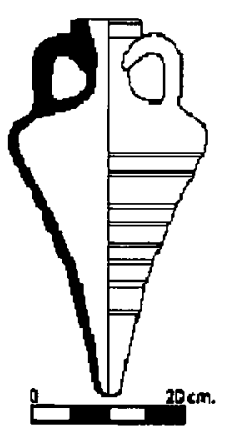

4

Figura 2. Diferentes dibujos de la $1^{a}$ ánfora de La Graciosa. 1: Dibujo de P. Cintas en Serra 1987: 428; 2: Pellicer 1970: 50, fig. 1; 3: García y Bellido 1970: 195, fig. 1; y 4: Beltrán 1970: 576, fig. 237, 2. 


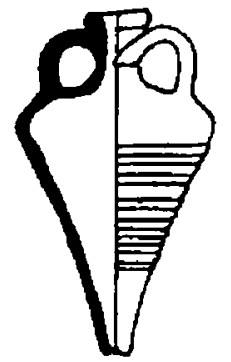

Ánfora $n^{\circ} 1$

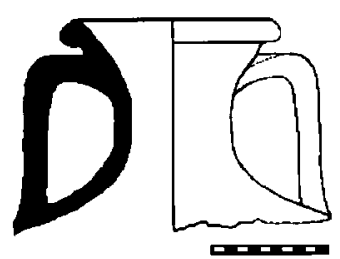

Ánfora $n^{\mathrm{n}} 10$

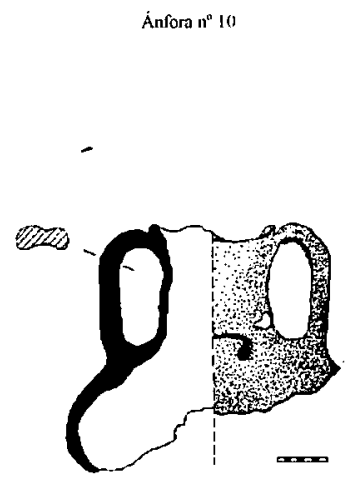

Ánfora $n^{\circ} 12$

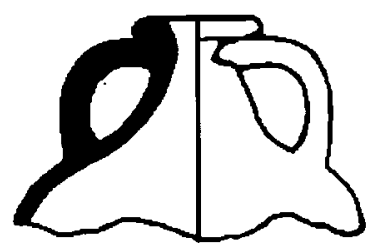

Änfora $n^{\circ} 2$

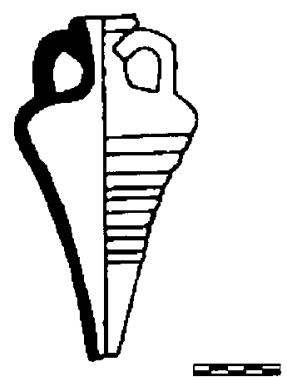

Ánfora $\mathbf{n}^{\circ} \mid 1$

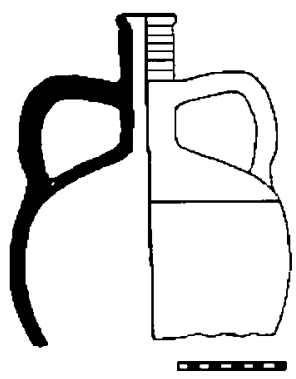

Ánfora $\mathrm{n}^{\circ} 13$

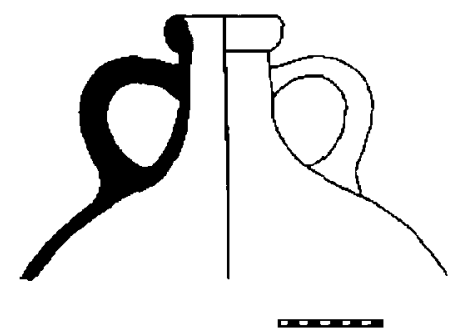

Ánfora $n^{\circ} 14$

Figura 3. Dibujos de ánforas aparecidas en aguas canarias según Escribano y Mederos 1996c: 92-98. 

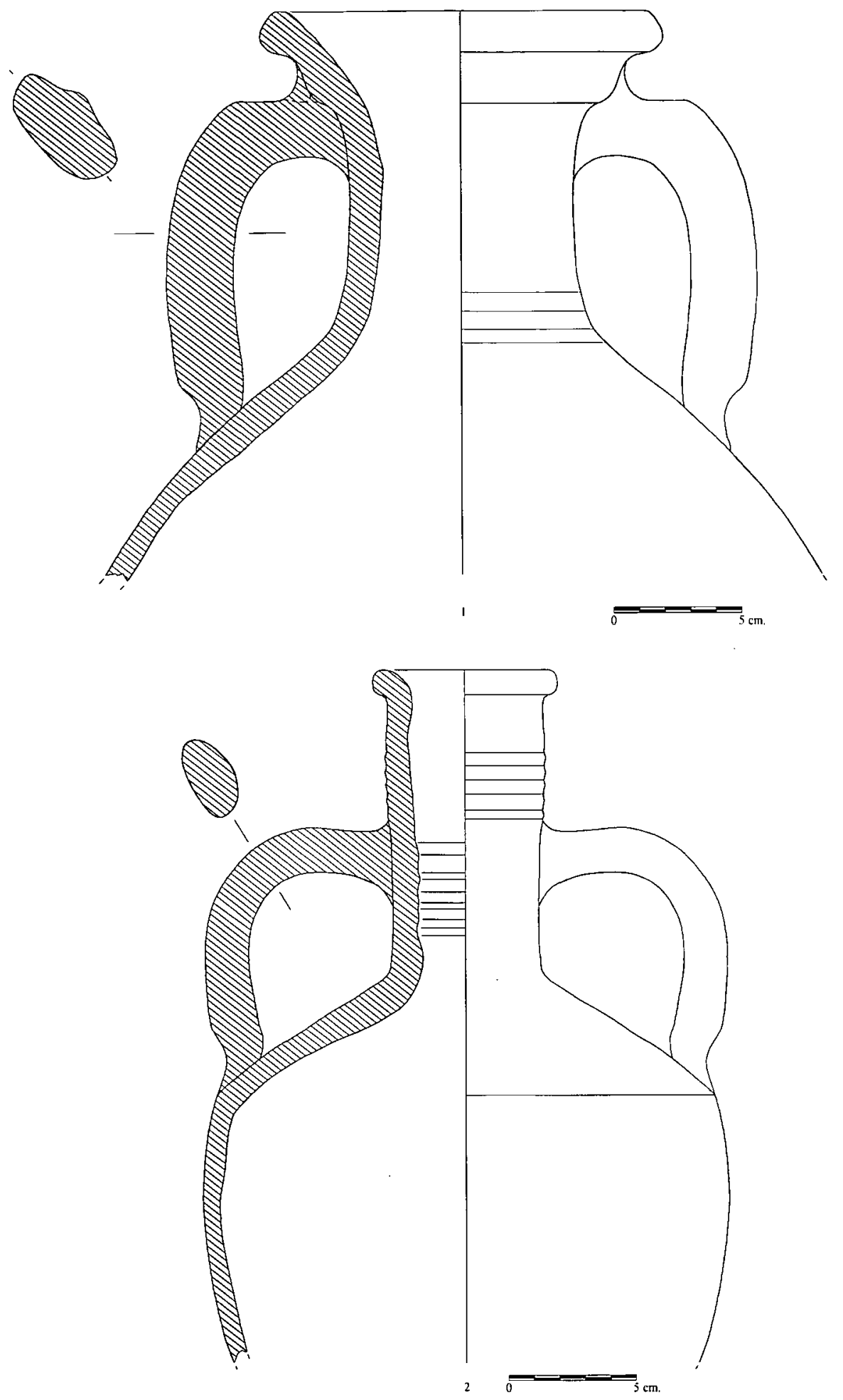

Figura 4. Ánforas aparecidas en aguas de Tenerife, Museo Arqueológico de Santa Cruz de Tenerife, $\mathrm{n}^{\circ} 1.069$ y 1.084. (Elaboración propia). 